\section{Aspectos epidemiológicos da leishmaniose tegumentar americana em Varzelândia, Minas Gerais, Brasil}

\author{
Epidemiological aspects of American tegumentary \\ leishmaniasis in Varzelândia, Minas Gerais, Brazil
}

Adriana Guimarães Nunes ${ }^{1}$

Edvá Vieira de Paula ${ }^{2}$

Roberto Teodoro ${ }^{3}$

Aluízio Prata ${ }^{1}$

Mario León Silva-Vergara ${ }^{1}$

\footnotetext{
1 Curso de Pós-graduação em Medicina Tropical e Infectologia, Universidade Federal do Triângulo

Mineiro, Uberaba, Brasil.

2 Fundação Nacional de Saúde, Montes Claros, Brasil.

3 Instituto de Ciências Biológicas, Universidade Federal de Minas Gerais, Belo Horizonte, Brasil.

Correspondência M. L. Silva-Vergara Curso de Pós-graduação em Medicina Tropical e Infectologia,

Universidade Federal do Triângulo Mineiro. C. P. 118, Uberaba, $M G$ 38001-970, Brasil. dip_fmtm@mednet.com.br
}

\section{Abstract}

To characterize an area of endemic leishmaniasis, aiming to test a candidate leishmania vaccine, a prospective epidemiological survey was implemented in 1999 in a rural area of Varzelândia, Minas Gerais, Brazil. From a total of 1,253 persons in 246 households, 1,170 were included, of whom 593 (50.6\%) were males and 662 (56.5\%) were under 21 years of age. A Montenegro intradermal test performed in 1,120 individuals and evaluated in 1,020 was reactive in 282 (27.6\%). Serological testing through indirect immunofluorescence and ELISA was performed in 970 individuals (82.9\%). Antibodies to Leishmania sp. were detected in 117 (13.1\%) and 170 (17.5\%), respectively, by the two tests. Cutaneous scars similar to those seen in American tegumentary leishmaniasis were found in 297 individuals (25.4\%), 282 of whom were submitted to the intradermal test, while only 168 (59.6) were reactive. Initial leishmaniasis prevalence of 5.8\% was recorded, and an annual leishmaniasis incidence rate of $4.6 \%$ was observed after one year of follow-up. The epidemiological characteristics observed in this location are suggestive of an endemic area with old colonization.

Leishmaniasis; Enzyme-Linked Immunosorbent Assay; Indirect Fluorescent Antibody Technique; Skin Tests

\section{Introdução}

As leishmanioses estão em expansão devido às graves modificações nos ecossistemas, sobretudo o desflorestamento para assentamentos populacionais, abertura de estradas, projetos de irrigação, construção de usinas hidrelétricas e urbanização desmedida, entre outros. Ademais, desnutrição, susceptibilidade genética, síndrome da imunodeficiência adquirida e resistência do parasita aos antimoniais pentavalentes em vários países, agravam a situação 1,2 .

O Brasil figura entre os cinco países que relatam mais de $90 \%$ dos casos de leishmaniose visceral e entre os sete com $90 \%$ dos casos de leishmaniose tegumentar no mundo 1,3. A urbanização das leishmanioses no país é um problema de saúde pública e, nas últimas décadas, ocorreram surtos em várias capitais 4,5. A incidência da doença aumentou substancialmente e o sub-registro de casos impede conhecer a magnitude do problema 1,6,7.

O controle das leishmanioses é prioridade da Organização Mundial da Saúde (OMS) e medidas como eliminação de vetores com inseticidas no domicilio, peridomicílio e colares impregnados para prevenir a infecção canina, além do sacrifício de cães infectados, não causaram o impacto esperado 8,9 . Uma vacina eficaz é outra alternativa, e diversos candidatos estão em fase de pesquisa clínica 10,11. 
O objetivo deste estudo foi caracterizar, epidemiologicamente, uma área de transmissão de leishmaniose tegumentar americana (LTA), na tentativa de testar candidato à vacina anti-leishmania.

\section{População e métodos}

O estudo foi realizado no Brejo do Mutambal, distrito rural do Município de Varzelândia, nordeste de Minas Gerais, Brasil. A população foi cadastrada, registrados dados demográficos e epidemiológicos e realizada avaliação clínica orientada para a detecção de lesões ativas e cicatriciais, sugestivas de LTA. A intradermorreação de Montenegro (IDRM) foi aplicada utilizando-se $0,1 \mathrm{ml}$ de solução de extrato antigênico de promastigotas mortas da Leishmania (Leishmania) amazonensis lote 656001 (Biobrás S.A., Belo Horizonte, Brasil), segundo técnica descrita 12 . A leitura foi realizada em 48 horas, e consideradas positivas as indurações $\geq 5 \mathrm{~mm} 13$. De cada indivíduo foram retirados $10 \mathrm{ml}$ de sangue por punção venosa, com sistema vacutainer. A imunofluorescência indireta (RIFI) com promastigotas de Leishmania (Leishmania) mexicana (MHOM/ $\mathrm{Br} / 60 / \mathrm{BH} 6)$ em crescimento exponencial em meio de LIT e o ELISA com o conjugado de antiimunoglobulina humana (Biomanguinhos, Rio de Janeiro, Brasil) foram realizados segundo técnicas previamente descritas 14 , no Instituto de Ciências Biológicas da Universidade Federal de Minas Gerais. Os soros reativos, a partir da diluição 1:40 e os valores de leitura superiores à média dos controles mais dois desvios-padrão, DO $>0,130$, foram considerados positivos, respectivamente, para ambos os testes.
Foram excluídas do inquérito crianças com menos de dois anos e gestantes. O projeto foi aprovado pelo Comitê de Ética em Pesquisa da Universidade Federal do Triângulo Mineiro. Realizada análise estatística com o programa Epi Info 6.04 (Centers for Disease Control and Prevention, Atlanta, Estados Unidos) e utilizado teste de proporção $\chi^{2}$ para comparação entre as variáveis e calculadas as razões de chance OR (IC95\%). Determinou-se um nível de significância estatística $\mathrm{p}<0,05$.

\section{Resultados}

Cadastrados 1.253 indivíduos, dos quais 1.170 distribuídos em 246 domicílios foram incluídos. Destes, 593 (50,6\%) masculinos e a maioria nasceu e habita na região. A idade variou de 1 a 84 anos, média de 26,1 e mediana de 20 anos. Quanto às ocupações, 455 (39\%) eram estudantes e 305 (26\%) lavradores.

Inicialmente, foram diagnosticados 68 (5,8\%) pacientes com LTA cutânea e mucosa e, após um ano de acompanhamento, registrados 45 $(4,6 \%)$ novos casos da doença.

A IDRM aplicada em 1.120 indivíduos, lida em 1.020 com 282 (27,6\%) dos casos positivos. Observada reatividade de $30,9 \%$ para o gênero masculino $(\mathrm{OR}=1,67$; IC95\%: 1,26-2,22; $\mathrm{p}=$ 0,002 ) e $24 \%$ para o feminino com diferença significativa para o gênero masculino em varias faixas etárias e OR com valores crescentes em relação direta com a idade (Tabela 1).

Cicatrizes cutâneas sugestivas de LTA foram observadas em 297 (25,4\%) indivíduos, dos quais $49,5 \%$ masculinos, localizadas nas extremidades em 227 (76,4\%) casos e, como lesão úni-

Tabela 1

Reatividade ao teste de Montenegro por faixa etária e gênero na população do Brejo do Mutambal, Varzelândia, Minas Gerais, Brasil.

\begin{tabular}{|c|c|c|c|c|c|c|c|c|c|c|}
\hline \multirow[t]{3}{*}{ Faixa etária (anos) } & \multirow[t]{3}{*}{ Indivíduos (n) } & \multicolumn{4}{|c|}{ Montenegro (+) } & \multicolumn{2}{|c|}{ Total } & \multirow[t]{3}{*}{$p^{*}$} & \multirow[t]{3}{*}{ OR } & \multirow[t]{3}{*}{ IC95\% } \\
\hline & & \multicolumn{2}{|c|}{ Masculino } & \multicolumn{2}{|c|}{ Feminino } & & & & & \\
\hline & & $\mathrm{n}$ & $\%$ & $\mathrm{n}$ & $\%$ & $\mathrm{n}$ & $\%$ & & & \\
\hline $2-11$ & 361 & 26 & 7,2 & 11 & 3,0 & 37 & 10,2 & 0,01 & 1,0 & \\
\hline $12-21$ & 301 & 35 & 11,6 & 23 & 7,6 & 37 & 19,2 & 0,09 & 2,09 & $1,3-3,3$ \\
\hline $22-31$ & 125 & 20 & 16,0 & 22 & 17,6 & 58 & 33,6 & 0,70 & 4,43 & $2,6-7,5$ \\
\hline $32-41$ & 95 & 22 & 23,1 & 12 & 12,6 & 42 & 35,8 & 0,05 & 4,62 & $3,1-6,8$ \\
\hline $42-51$ & 89 & 22 & 24,7 & 19 & 21,3 & 34 & 46,0 & 0,59 & 7,48 & $4,2-13,2$ \\
\hline $52-61$ & 70 & 16 & 22,8 & 22 & 31,4 & 41 & 54,2 & 0,25 & 8,6 & $5,7-13,2$ \\
\hline $62-71$ & 44 & 11 & 25,0 & 5 & 11,3 & 38 & 36,3 & 0,09 & 5,0 & $2,3-10,6$ \\
\hline$\geq 72$ & 35 & 8 & 22,7 & - & - & 16 & 45,6 & 0,35 & 7,3 & $3,2-16,5$ \\
\hline Total & 1.120 & 160 & 14,2 & 122 & 10,8 & 282 & 27,6 & & & \\
\hline
\end{tabular}


ca, em 186 (62,6\%). Daqueles com cicatriz, 282 realizaram a IDRM, com reatividade em 168 $(59,6 \%)$ dos casos; quando distribuídos por faixa etária, o coeficiente kappa mostrou correlação insatisfatória (Tabela 2).

A pesquisa de anticorpos anti-leishmania em 970 (82,9\%) indivíduos, pelo RIFI e ELISA, mostrou positividade em $127(13,1 \%)$ e 170 $(17,5 \%)$ casos, respectivamente, e concordância de $76 \%$ entre ambos os testes. Não houve diferença entre os gêneros (Tabela 3).

Os indivíduos com RIFI e ELISA positivos e IDRM reativa mostraram concordância em $41,3 \%$ e $39,2 \%$ dos casos, respectivamente. Igualmente, os positivos para esses testes e com cicatrizes cutâneas sugestivas de LTA e, quando as reações sorológicas eram negativas, havia cicatriz cutânea em somente $6,4 \%$ dos casos.

\section{Comentários}

O inquérito foi realizado numa área com características de colonização antiga, onde há pouca mata a ser derrubada e em seu lugar as diversas atividades agropecuárias estão associadas à transmissão permanente dessa infecção 2,15 . Isso justifica a presença de cicatrizes cutâneas sugestivas de LTA em $25 \%$ dos habi-

Tabela 2

Intradermorreação de Montenegro (IDRM) em 282 indivíduos com cicatriz cutânea semelhante às causadas pela leishmaniose tegumentar em área endêmica, no Brejo do Mutambal, Varzelândia, Minas Gerais, Brasil.

\begin{tabular}{|c|c|c|c|c|c|c|c|c|}
\hline \multirow{2}{*}{$\begin{array}{l}\text { Faixa etária } \\
\text { (anos) }\end{array}$} & \multirow[t]{2}{*}{ Indivíduos (n) } & \multicolumn{3}{|c|}{ Cicatriz cutânea } & \multicolumn{4}{|c|}{ IDRM positivo } \\
\hline & & $\mathrm{n}$ & $\%$ & OR & IC95\% & $n$ & $\%$ & $p^{*}$ \\
\hline $2-11$ & 361 & 49 & 17,3 & 1,0 & - & 24 & 49,0 & 0,002 \\
\hline $12-21$ & 301 & 91 & 32,2 & 3,7 & $2,6-5,1$ & 42 & 46,1 & 0,0001 \\
\hline $22-31$ & 125 & 32 & 11,3 & 2,2 & $1,3-3,7$ & 22 & 68,7 & 0,12 \\
\hline $32-41$ & 95 & 31 & 11,0 & 3,1 & $1,8-5,4$ & 20 & 64,5 & 0,07 \\
\hline $42-51$ & 89 & 32 & 11,3 & 3,5 & $2,0-6,2$ & 26 & 81,2 & 0,33 \\
\hline $52-61$ & 70 & 30 & 10,6 & 4,8 & $2,6-8,6$ & 23 & 76,6 & 0,16 \\
\hline $62-71$ & 44 & 10 & 3,5 & 1,8 & $0,8-4,2$ & 7 & 70,0 & 0,41 \\
\hline$>72$ & 35 & 7 & 2,5 & 1,6 & $0,6-4,1$ & 4 & 57,1 & 0,32 \\
\hline Total & 1.120 & 282 & 27,6 & & & 168 & 59,5 & \\
\hline
\end{tabular}

Tabela 3

Distribuição dos indivíduos reativos à imunofluorescência indireta e ELISA por faixa etária e gênero no Brejo do Mutambal, Varzelândia, Minas Gerais, Brasil.

\begin{tabular}{|c|c|c|c|c|c|c|c|c|c|}
\hline \multirow{3}{*}{$\begin{array}{l}\text { Faixa etária } \\
\text { (anos) }\end{array}$} & \multirow[t]{3}{*}{ Indivíduos (n) } & \multicolumn{4}{|c|}{ RIFI positivo } & \multicolumn{4}{|c|}{ ELISA positivo } \\
\hline & & \multicolumn{2}{|c|}{ Masculino } & \multicolumn{2}{|c|}{ Feminino } & \multicolumn{2}{|c|}{ Masculino } & \multicolumn{2}{|c|}{ Feminino } \\
\hline & & $\mathrm{n}$ & $\%$ & $n$ & $\%$ & $\mathrm{n}$ & $\%$ & $\mathrm{n}$ & $\%$ \\
\hline $2-11$ & 312 & 22 & 17,3 & 17 & 13,3 & 20 & 11,7 & 10 & 5,8 \\
\hline $12-21$ & 261 & 11 & 8,6 & 17 & 13,3 & 18 & 10,5 & 22 & 12,9 \\
\hline $22-31$ & 108 & 8 & 6,2 & 12 & 9,4 & 12 & 7,0 & 17 & 10,0 \\
\hline $32-41$ & 82 & 6 & 4,7 & 5 & 3,9 & 14 & 8,2 & 08 & 4,7 \\
\hline $42-51$ & 77 & 3 & 2,3 & 5 & 3,9 & 6 & 3,5 & 10 & 10,0 \\
\hline $52-61$ & 61 & 4 & 3,1 & 5 & 3,9 & 8 & 4,7 & 8 & 4,7 \\
\hline $62-71$ & 38 & 6 & 6,2 & 3 & 2,3 & 6 & 3,5 & 6 & 3,5 \\
\hline$\geq 72$ & 30 & 0 & - & 3 & 2,3 & 2 & 1,1 & 3 & 1,7 \\
\hline Subtotal & & 60 & 47,2 & 67 & 52,7 & 86 & 51,5 & 84 & 49,0 \\
\hline Total & 970 & 127 & 13,0 & & & & & 170 & 17,5 \\
\hline
\end{tabular}


tantes, a prevalência de 5,8\% de casos e a incidência de $4,3 \%$ após um ano.

Entre 580 indivíduos examinados, numa área com características semelhantes à de Varzelândia, foram encontrados $23,7 \%$ com cicatrizes 16. Neste estudo, foram vistos $297(25,4 \%)$ casos com lesões cicatriciais. Destes, 168 (59,6\%) com IDRM positiva, em contraste com $27,6 \%$ observados na população geral da área. Essas lesões, quando bem caracterizadas, podem servir como marcador clínico-epidemiológico de LTA mais sensível e concordante com os testes sorológicos do que a IDRM, embora outras condições como traumatismos, queimaduras e piodermites etc. possam deixar cicatrizes semelhantes.

A alta prevalência de reatividade ao antígeno de Montenegro (27,6\%) dos casos tem sido assinalada em outras áreas onde LTA é endêmica e não está necessariamente associada a lesões ativas ou cicatriciais. Mesmo excluindo essas lesões, ainda houve $13 \%$ de indivíduos com IDRM positiva. Como é sabido, infecções subclínicas e outras patologias endêmicas coexistentes podem positivá-la 17 .

Os testes RIFI e ELISA foram positivos em $13,1 \%$ e $17,5 \%$ indivíduos, respectivamente. Tais resultados devem ser interpretados com cautela, haja vista que podem positivar em pacientes com lesões ativas, podem indicar infecção recente na ausência de lesões ou ser a conseqüência de reinfecções subclínicas 18. É interessante ressaltar que, neste estudo, a coexistência de lesões cicatriciais semelhantes às de
LTA, foi constatada em $85,7 \%$ dos casos que tinham ambos os testes sorológicos reativos. $\mathrm{O}$ achado de parasitas em cicatrizes antigas, como estímulo antigênico, também explica a presença de anticorpos nesses indivíduos 19. Por outro lado, infecção por Trypanosoma cruzi pode dar reação cruzada em indivíduos co-infectados com Leishmania sp. 17.

A observação de cicatrizes cutâneas, úlceras ativas e Montenegro positiva entre crianças, adolescentes e mulheres, sugere transmissão domiciliar ou peridomiciliar, corroborada pela localização das moradias próximas de pedreiras e a convergência do vetor mais adaptado e de animais domésticos, potenciais reservatórios da infecção, principais características da área avaliada e semelhante a outros estudos 9,16,20.

Estes achados são comuns quando Leishmania (Viannia) braziliensis é a espécie endêmica 21. No Brasil, é a espécie mais prevalente nas áreas de colonização antiga com profundas modificações no ecossistema como aquelas observadas nessa área em estudo 15,21. Ademais, a presença de pacientes com formas mucosas e o predomínio de lesões cutâneas ativas e cicatriciais únicas corroboram essa etiologia.

Os resultados descritos caracterizam esse local como área endêmica de leishmaniose, onde há convergência dos fatores epidemiológicos que favorecem a transmissão ativa, há várias décadas, e pode ser passível de intervenção sob diferentes aspectos, com vistas ao seu controle.

\section{Resumo}

Para caracterizar uma área endêmica de leishmaniose tegumentar americana susceptivel de intervenção com candidato à vacina anti-leishmania, foi iniciado em 1999 estudo longitudinal em área rural do Município de Varzelândia, Minas Gerais, Brasil. Inicialmente, foram cadastrados 1.253 indivíduos distribuídos em 246 domicílios, dos quais, 1.170 concordaram em participar do trabalho. Desses, 593 (50,6\%) eram masculinos e 662 (56,5\%), menores de 21 anos. A intradermorreação de Montenegro foi realizada em 1.120 indivíduos, lida em 1.020 dos casos, e observada reatividade em 282 (27,6\%) deles. Anticorpos anti-leishmania foram testados mediante imunofluorescência indireta e teste ELISA em 970 (82,9\%) indivíduos, obtendo-se positivi- dade, respectivamente, em 127 (13,1\%) e 170 (17,5\%) para os dois testes. Na avaliação inicial, foram observados 297 (25,4\%) indivíduos com cicatrizes cutâneas semelhantes às deixadas pela doença. Desses, 282 realizaram a intradermorreação que foi positiva em168 (59,61\%). A prevalência de casos de leishmaniose tegumentar no início do estudo foi de 5,8\% e, depois do primeiro ano, observou-se incidência anual de 4,6\% de casos. As características epidemiológicas observadas nesse local sugerem tratar-se de uma área endêmica de colonização antiga.

Leishmaniose; ELISA; Técnica Indireta de Fluorescência para Anticorpo; Testes Cutâneos 


\section{Colaboradores}

Todos os co-autores participaram, integral ou parcialmente, em cada uma das fases de desenvolvimento do presente projeto. O trabalho de campo foi desenvolvido por A. G. Nunes, M. L. Silva-Vergara e E. V Paula. Os testes sorológicos foram realizados por R. Teodoro. O manuscrito foi redigido por A. G. Nunes, A. Prata e M. L. Silva-Vergara.

\section{Referências}

1. Desjeux P. Leishmaniasis: current situation and new perspectives. Comparative immunology. Microbiol Infect Dis 2004; 27:305-18.

2. Grimaldi Jr. G, Tesh RB, Mc Mahon-Pratt D. A review of the geographical distribution and epidemiology of leishmaniasis in the New World. Am J Trop Med Hyg 1989; 41:687-725.

3. Ashford R. The leishmaniasisas emerging and reemerging zoonosis. Int J Parasitol 2000; 30:126981.

4. Marzochi MCA, Marzochi KBF. Tegumentary and visceral leishmaniasis in Brazil. Emerging anthropozoonosis and possibilities for their control. Cad Saúde Pública 1994; 10:359-75.

5. Passos VMA, Falcão AL, Marzochi MCA, Gontijo CMF, Dias ES, Barbosa-Santos EGO; Epidemiological aspects of American cutaneous leishmaniasis in a periurban area of the metropolitan region of Belo Horizonte, Minas Gerais, Brazil. Mem Inst Oswaldo Cruz 1993; 88:103-10.

6. Fundação Nacional de Saúde/Centro Nacional de Epidemiologia. Evolução temporal das doenças de notificação compulsória no Brasil de 1980 a 1998. Boletim Epidemiológico 1999; Edição especial.

7. Centro Nacional de Epidemiologia, Fundação Nacional de Saúde. Manual de controle da leishmaniose tegumentar americana. Brasília: Centro Nacional de Epidemiologia, Fundação Nacional de Saúde; 1997.

8. Courtenay O, Quinnell RJ, Garcez LM, Shaw JJ, Dye C. Infectiousness in a cohort of Brazilian dogs: why culling fails to control visceral leishmaniasis in areas of high transmission. J Infect Dis 2002; 186: 1314-20.

9. Campbell-Lendrum D, Dujardin JP, Martinez E, Feliciangeli MD, Perez JE, Passerat de Silans LNM, et al. Domestic and peridomestic transmission of American cutaneous leishmaniasis: changing epidemiological patterns present new control opportunities. Mem Inst Oswaldo Cruz 2001; 96:159-62.

10. Armijos RX, Weigel MM, Romero L, Garcia V, Salazar J. Field trial of a vaccine against new world cutaneous leishmaniasis in an at-risk child population: how long does protection last? J Infect Dis 2003; 187:1959-61.

11. Handman E. Leishmaniasis: current status of vaccine development. Clin Microbiol Rev 2001; 14: 229-43.

12. Mayrink W, Williams $P$, Coelho MV, Dias M, Martins VA, Magalhães PA, et al. Epidemiology of

\section{Agradecimentos}

Este estudo recebeu auxílio financeiro do Conselho Nacional de Desenvolvimento Científico e Tecnológico e da Fundação Nacional de Saúde.

dermal leishmaniasis in Rio Doce Valley, State of Minas Gerais, Brazil. Ann Trop Med Parasitol 1979; 73:123-37.

13. Sokal JE. Measurement of delayed sign test responses. N Engl J Med 1975; 293:501-2.

14. Camargo ME, Rebonato C. Cross reactivity in fluorescence tests for trypanosoma and leishmania antibodies. A simple inhibition procedure to ensure specific results. Am J Trop Med Hyg 1969; 18: 500-5.

15. Grimaldi Jr. G, Tesh RB. Leishmaniasis of the New World: current concepts and implications for future research. Clin Microbiol Rev 1993; 6:230-50.

16. Ampuero J, Urdaneta M, Macêdo VO. Factores de riesgo para la transmisión de leiishmaniasis cutánea en niños de 0 a 5 años en un área endémica de Leishmania (Viannia) braziliensis. Cad Saúde Pública 2005; 21:161-70.

17. Vexenat AC, Santana JM, Teixeira ARL. Cross reactivity of antibodies in human infections by the kinetplastid protozoa Trypanosoma cruzi, Leishmania chagasi and Leishmania braziliensis. Rev Inst Med Trop São Paulo 1996; 38:177-85.

18. Marzochi MCA, Coutinho SG, Sabroza PC, Souza WJS. Reação de imunofluorescência indireta e intradermorreação de Montenegro para leishmaniose tegumentar americana em moradores da área de Jacarepaguá (Rio de Janeiro). Estudo comparativo dos resultados observados entre 1974 e 1978. Rev Inst Med Trop São Paulo 1980; 22:149-55.

19. Schubach A, Marzochi MCA, Cuzzi-Maya T, Oliveira AV, Araújo ML, Oliveira ALC, et al. Cutaneous scars in American tegumentary leishmaniasis patients. A site of Leishmania Viannia braziliensis persistence and viability 11 years after antimonial therapy and clinical cure. Am J Trop Med Hyg 1998; 58:824-7.

20. Falqueto A. Especificidade alimentar de flebotomíneos em duas áreas de leishmaniose tegumentar no Estado do Espírito Santo, Brasil. Rev Soc Bras Med Trop 1997; 30:531-2.

21. Marsden PD. Mucosal leishmaniasis ("Espundia" Escomel 1911). Trans R Soc Trop Med Hyg 1986; 80: 859-76.

Recebido em 01/Abr/2005

Versão final reapresentada em 30/Ago/2005

Aprovado em 02/Fev/2006 